\title{
Muséologies
}

Les cahiers d'études supérieures

\section{Tout nouveau, tout ado!}

\section{Chantal Steegmuller}

Volume 2, numéro 1, octobre 2007

URI : https://id.erudit.org/iderudit/1033604ar

DOI : https://doi.org/10.7202/1033604ar

Aller au sommaire du numéro

Éditeur(s)

Association Québécoise de Promotion des Recherches Étudiantes en Muséologie (AQPREM)

ISSN

1718-5181 (imprimé) 1929-7815 (numérique)

Découvrir la revue

Citer ce document

Steegmuller, C. (2007). Tout nouveau, tout ado! Muséologies, 2(1), 156-159.

https://doi.org/10.7202/1033604ar d'utilisation que vous pouvez consulter en ligne.

https://apropos.erudit.org/fr/usagers/politique-dutilisation/ 


\section{Tout nouveau tout ado!}

[Par Chantal Steegmuller] 
Les adolescents ne constituent pas un public "naturel " des institutions muséales, puisqu'ils les fréquentent généralement dans un cadre scolaire ou familial ${ }^{[1]}$. Pourtant, si la relation musée-adolescents a longtemps été négligée par les professionnels de musée et les chercheurs, elle est aujourd'hui sous les feux de la rampe. En effet, la reconnaissance du public adolescent comme un public à part entière, ni enfant ni adulte, est depuis plusieurs années considérée dans de nombreux champs disciplinaires, auxquels la muséologie ne fait pas exception. Le musée, au même titre que l'école et la communauté, reconnaît l'importance de proposer aux adolescents un environnement stimulant, qui favorise leur apprentissage et leur développement cognitif et social ${ }^{[2]}$.

Ce défi, le Centre Georges-Pompidou à Paris a décidé de le relever. En effet, le Centre inaugurera à l'automne 2008 un tout nouvel espaceatelier entièrement consacré à ce public.

\section{Le projet}

Le trentième anniversaire du Centre Pompidou fut l'occasion pour toute l'équipe de la programmation jeune public de réfléchir à la façon dont ce service, reconnu pour son expérience et son savoir-faire en matière de médiation, pouvait prendre en main celle destinée aux adolescents.

L'objectif de cet atelier, selon Patrice Chazottes, responsable de la programmation jeune public et chef du projet, est double. D'abord, démontrer que l'on peut bel et bien concevoir des activités pour et avec ce public; puis offrir l'occasion aux adolescents de s'ouvrir aux diverses formes que peut prendre l'art contemporain, grâce à une interaction directe avec les artistes. L'intention est ainsi de faire de cet espace, créé en collaboration avec des étudiants de l'École nationale supérieure de création industrielle (ENSCI) ${ }^{[3]}$, un lieu d'activités que les adolescents fréquenteront de façon régulière.
[1]

DAIGNAULT, Lucie.

"Le passé et l'avenir des

"ados" au Musée. La Pratique

Muséale. Québec", Les cahiers

du Musée de la Civilisation,

2001. p. 9.

\section{[2]}

SOUCY, Brenda.

Enquête auprès d'adolescents de la région de Montréal et de ses proches banlieues sur leurs connaissances et perceptions des musées, pratiques de visites, préférences et intérêts pour différents types de contextes et de projets muséaux. Thèse de doctorat en psychologie, Université du Québec à Montréal, 1999, p. 6 et 8.
[3]

La collaboration avec les étudiants de l'ENSCl se fait tant sur le plan de l'aménagement de l'espace que ceux du concept des activités et de la communication. 


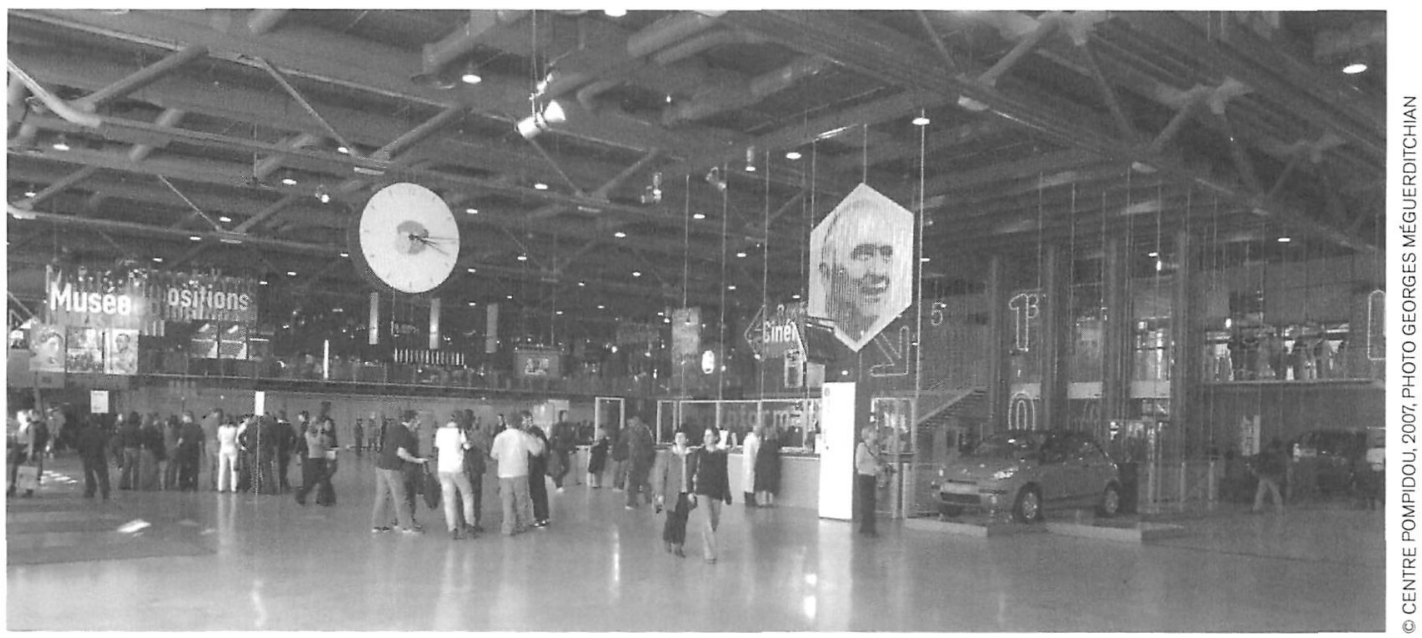

\section{Les activités}

Dans cet espace pluridisciplinaire de 400 mètres carrés se côtoieront ainsi les arts plastiques, le cinéma, la musique, la danse, la vidéo ainsi que les nouvelles technologies. L'atelier s'organisera autour d'un thème central ${ }^{[4]}$, se développant sur un ou deux mois, ponctué de moments forts : manifestations sportives, événements musicaux, fins de semaine "carte blanche" animées par des personnalités connues. Chaque activité sera conçue de manière à permettre aux adolescents d'être à la fois spectateurs et acteurs au sein de cet espace. Puisque les artistes ne seront pas présents en permanence, une grande place sera octroyée au dialogue entre les adolescents : artistes, jeunes créateurs, étudiants de divers secteurs pourront alors mener la médiation in situ.

\section{Le public et la promotion}

L'atelier sera plus spécifiquement destiné au public adolescent de la région francilienne, ouvert en semaine au public scolaire, ainsi que les mercredis, les fins de semaine et durant les vacances scolaires au public individuel.

Comme c'est un fait connu qu'il est difficile d'attirer les adolescents au musée, une attention toute particulière sera accordée à la promotion de cet espace. Des collaborations avec les institutions scolaires et les départements ${ }^{[5]}$ limitrophes de la région parisienne, ainsi que des partenariats avec différents médias - radio et télévision -seront établis. Le streetmarketing sera également utilisé pour rejoindre les adolescents dans leurs lieux de vie : les magasins, les parcs, les centres
[4]

L'activité inaugurale,

"Habitez vos habits", sera réalisée avec la participation de l'École supérieure des arts appliqués Duperré, à Paris. D'autres thématiques sont déjà envisagées : une activité sur l'émotion sera abordée avec la collaboration d'un chorégraphe et d'un plasticien un laboratoire numérique sera monté pour présenter diverses technologies (photographie numérique, logiciels) en collaboration avec un collectif d'artistes.

[5]

Un partenariat avec les départements possédant les compétences nationales par rapport aux collèges et aux transports permettrait, d'une part, de proposer aux adolescents des facilités de transport pour la visite du
Centre Pompidou et, d'autre part, de mener des actions in situ dans les différents départements. 
sportifs ou encore les murs de graffiti reconnus. De plus, un site Internet permettra de présenter les activités à venir, ainsi que les vidéos des événements passés.

La fidélisation du public visé est également envisagée par la création d'une carte de membre permettant aux adolescents d'entrer directement dans l'atelier sans passer par la caisse ${ }^{[6]}$. Cette carte leur permettrait aussi de recevoir les infolettres directement dans leur boîte courriel ou sur leur téléphone portable.

\section{L'impact de l'atelier sur le milieu muséal}

Pour que l'image que se font les adolescents des musées change, il faudrait d'abord, selon
Patrice Chazottes, que les musées modifient leur approche. C'est pour cette raison qu'une équipe de recherche sera créée pour évaluer cet espace, afin de documenter les activités et ainsi proposer une réflexion et un regard croisé sur le projet.

L'atelier ne changera peut-être que l'image du Centre Pompidou, mais il donnera - éventuellement - aux autres musées un argument pour changer à leur tour (et à leur manière) leur programmation ado!

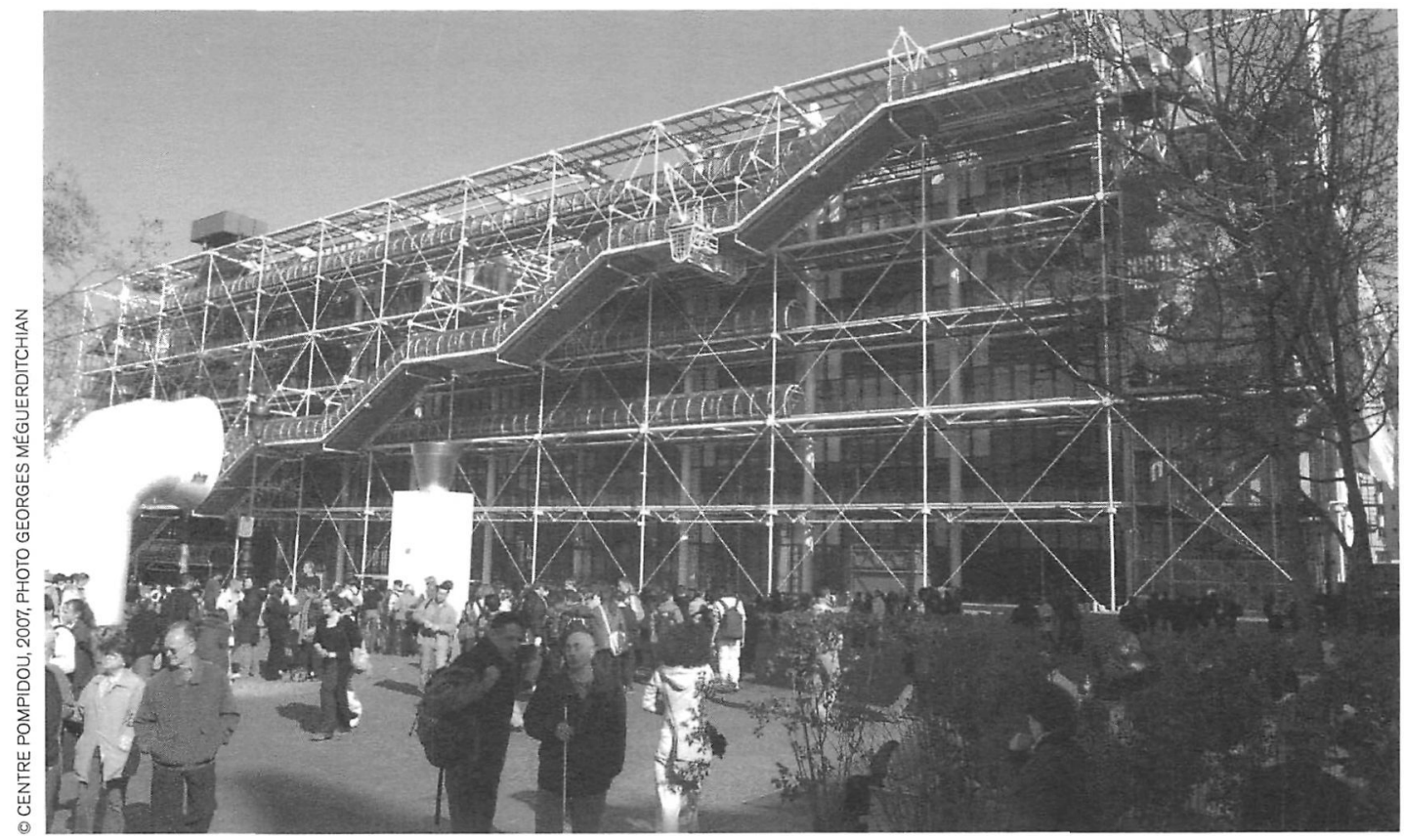

[6]

Les moins de 18 ans entrent de toute façon gratuitement au musée, mais doivent retirer un billet exonéré. 